\title{
MODELAGEM E SIMULAÇÃO FLUIDODINÂMICA DE UM SECADOR INDUSTRIAL DE PVC USANDO A TEORIA CINÉTICA DE FLUXO GRANULAR
}

\author{
W. R. de ARAUJO ${ }^{1}$, A. T. PEREIRA NETO ${ }^{1}$, A. R. TACIDELLI ${ }^{2}$, e J. J. N. ALVES ${ }^{1}$ \\ ${ }^{1}$ Universidade Federal de Campina Grande, Departamento de Engenharia Química \\ ${ }^{2}$ BRASKEM Petroquimica S. A., Engenharia de Processos, Unidade de Vinilicos \\ E-mail para contato: jailson@ deq.ufcg.edu.br
}

\begin{abstract}
RESUMO - Os secadores de leito fluidizado são utilizados no processo de produção de Policloreto de Vinila (PVC). Um dos problemas operacionais destes equipamentos é a possibilidade de formação de aglomerados e montanhas de PVC devido à má fluidização do leito. Com o objetivo de prever a formação destes aglomerados, o escoamento no secador industrial de PVC é estudado, neste trabalho, por meio de técnicas de fluidodinâmica computacional (CFD), as quais permitem a avaliação do comportamento da partícula e do fluido utilizado para a secagem, bem como a interação entre estes. A Teoria Cinética do Escoamento de Materiais Granulares (KTGF) foi testada para considerar as interações partícula-partícula. A formação de aglomerados foi prevista com um modelo invíscido para a fase particulada, mas esta característica não foi prevista com o modelo da KTGF. O software ANSYS CFX foi utilizado como ferramenta para as simulações.
\end{abstract}

\section{INTRODUÇÃO}

Secadores de leito fluidizado são vastamente utilizados na indústria química, particularmente no processo de produção de resinas de PVC, onde a etapa final do processo é a secagem das partículas do polímero. Um dos problemas associados à operação destes secadores de PVC é a possibilidade de formação de aglomerados de partículas de PVC (clusters), dependendo das condições operacionais. A previsão desta má fluidização é fundamental para a minimização do problema. Estes clusters normalmente estão presentes neste tipo de equipamento devido à formação de zonas de recirculação. Estes aglomerados são indesejáveis, pois reduzem o rendimento do processo de secagem.

Existem alguns trabalhos na literatura voltados à modelagem cinética e otimização do processo de secagem de PVC (Araujo et al., 2010; Tacidelli et al., 2012). Outros trabalhos são voltados à modelagem da fluidodinâmica do secador. Duas abordagens têm sido normalmente utilizadas para a modelagem em CFD dos fluxos gasoso e sólido: a abordagem Euleriana-Lagrangiana e a abordagem Euleriana-Euleriana. Na abordagem Euleriana-Lagrangiana, a trajetória de Lagrange para o estudo do movimento das partículas individuais é acoplada com a formulação Euleriana para o gás (Assari et al., 2013). Na abordagem Euleriana-Euleriana o conjunto de partículas é tratado como uma fase fluida continua e as equações de conservação médias para esta fase precisam ser obtidas (Alves, 1998). O 


\section{9 a 22 de outubro de 2014 \\ Florianópolis/SC}

modelo mais simples para a fase particulada trata-a como fluido invíscido. Outro modelo é o da teoria cinética do escoamento de materiais granulares (KTGF), que se baseia no pressuposto de que a velocidade de distribuição individual das partículas, entre um grande número de partículas dentro de um conjunto do volume, pode ser representada pela distribuição dos seus pontos de velocidade. Assim como a teoria cinética clássica, a teoria cinética do escoamento granular assume "caos molecular". Isto implica que a distribuição de velocidades das partículas para todas as partículas é isotrópica e que as velocidades de duas partículas envolvidas numa colisão não estão correlacionadas (Goldschmidt et al., 2002). van der Hoef et al. (2005) mencionaram que a vantagem deste modelo é que o mesmo pode prever o comportamento do escoamento gás-sólido em escalas de tamanho natural e, por conseguinte, estes modelos são amplamente utilizados em simuladores de escoamento comerciais.

A temperatura granular para o modelo da teoria cinética pode ser especificada ou calculada de forma algébrica pela consideração de equilíbrio local no modelo da equação de transporte ou ainda baseada no modelo com zero equação, o qual utiliza o modelo algébrico simples de Ding e Gidaspow (ANSYS, 2011).

Neste trabalho, buscou-se identificar um modelo capaz de prever a formação de aglomerados de partículas e proporcionar, no futuro, a utilização do modelo para identificar novas condições de operação ou modificações na geometria do secador que minimizem a formação destes aglomerados.

\section{ESTUDO DE CASO}

No secador em estudo, o sólido entra pela parte superior e o gás por distribuidores na parte inferior do secador. O PVC sai por uma lateral do secador e o gás por cima, porém uma pequena fração de sólidos é arrastada pela saída superior. A região do leito com maior presença das duas fases situa-se próxima ao fundo.

A simulação tomou como base a abordagem Euleriana-Euleriana do escoamento multifásico no interior do secador, onde o modelo foi resolvido considerando a interação das duas fases.

Procurou-se estudar uma modelagem tridimensional, bifásica e isotérmica, bem como testar o modelo cinético do escoamento de materiais granulares.

O modelo matemático do processo baseia-se nos princípios de conservação da massa e de quantidade de movimento para a fase gasosa e a particulada (Alves, 1998; Coutinho, 2003).

A viscosidade efetiva do gás pode ser estimada partindo de qualquer modelo de turbulência (Nieuwland et al., 1996). O modelo de turbulência $k-\varepsilon$ foi utilizado para a fase gás. Para a interação entre a fase contínua e a particulada foi considerado o modelo de Schiller Naumann. A teoria cinética do escoamento de materiais granulares foi testada para a fase particulada, bem como um modelo invíscido para a mesma.

Utilizou-se, no estudo, o secador representado pela Figura 1, que mostra as regiões de entrada e saída do gás e do sólido. O gás entra pela região inferior e desempenha um fluxo ascendente, 
enquanto o sólido entra pela lateral. Existe uma caixa coletora de sólidos na face oposta à entrada destes.

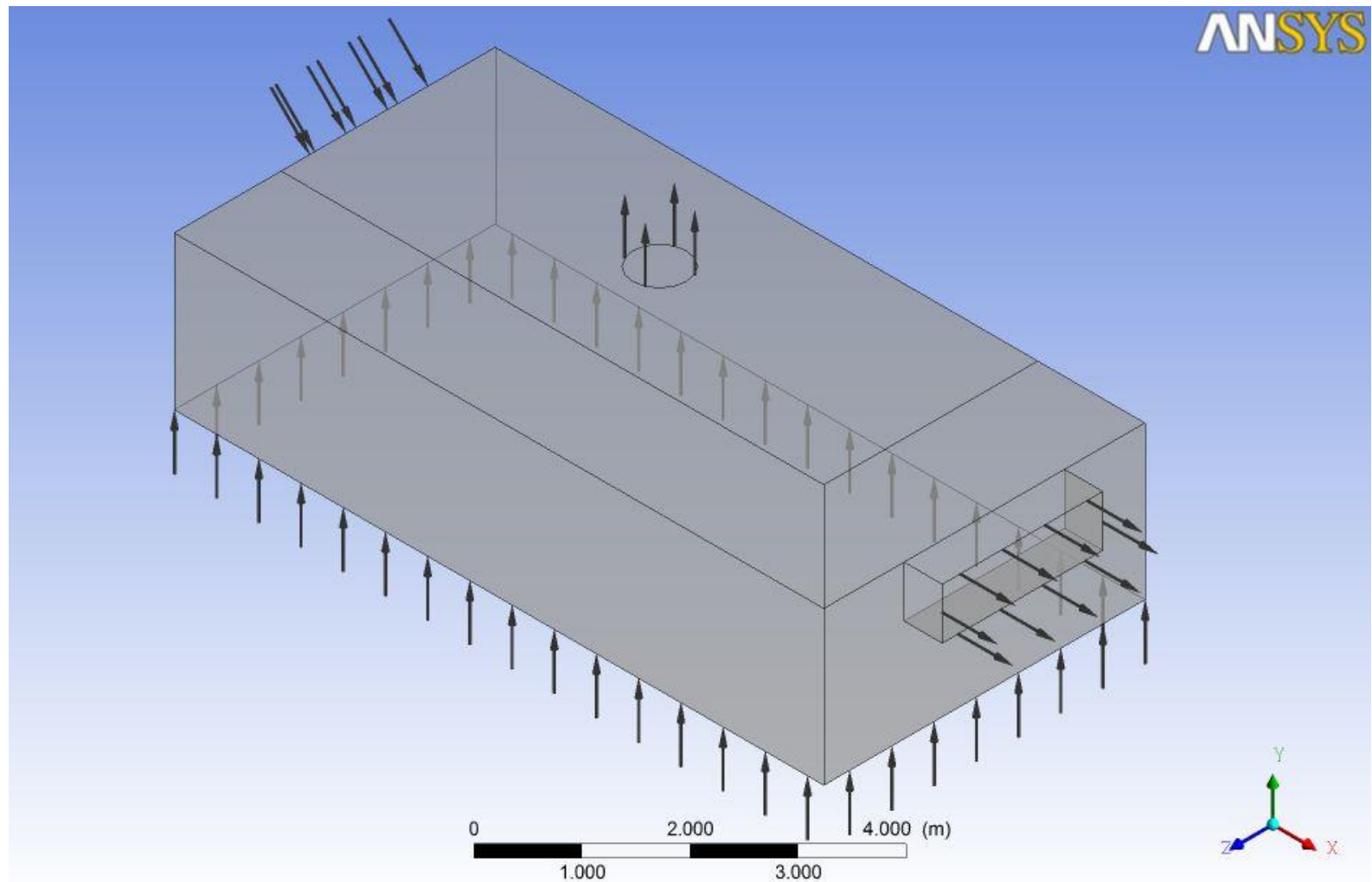

Figura 1 - Representação esquemática do equipamento utilizado no estudo.

As condições reais de operação do secador industrial estão disponíveis em Tacidelli et al. (2012). Utilizou-se a estratégia de falso transiente, que consiste em resolver as equações com o termo transiente, no entanto sem forçar a convergência completa para cada instante de tempo, até atingir o estacionário.

\section{RESULTADOS}

A Figura 2 mostra a fração volumétrica de PVC ao longo do plano XY em z $=2 \mathrm{~m}$ (próximo ao meio do equipamento). A formação do leito fluidizado no processo de secagem do PVC foi prevista com o modelo invíscido. A altura do leito prevista atingiu um patamar ligeiramente inferior ao vertedouro de saída de sólidos. 


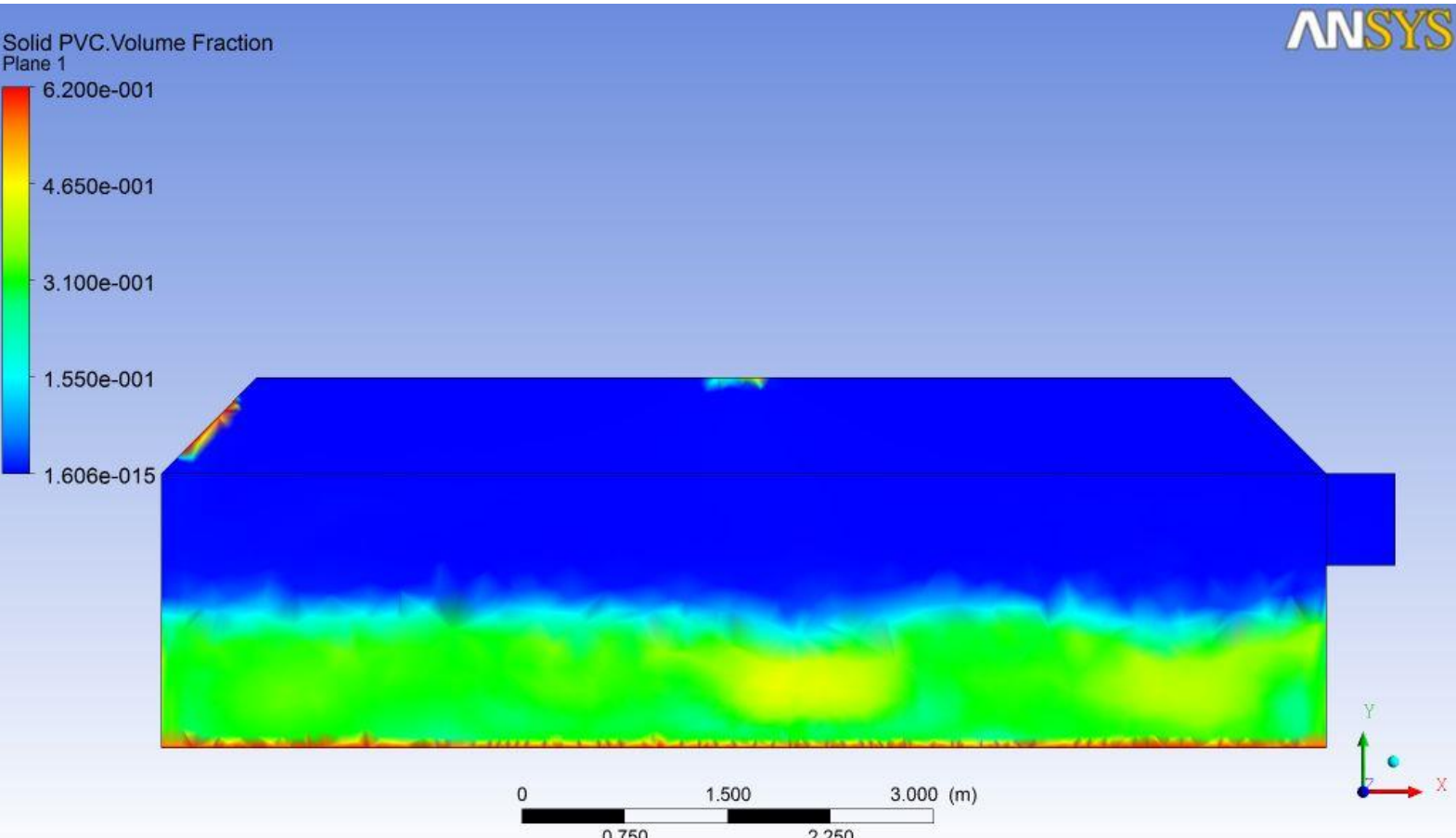

Figura 2 - Fração volumétrica de PVC no plano XY em z = 2 m para o modelo invíscido.

De acordo com a Figura 2, nota-se a existência de uma região de alta concentração de sólidos, na porção inferior do secador, com aproximadamente $62 \%$ de PVC, o que está de acordo com o observado na planta industrial.

A Figura 3 mostra uma pequena fração de PVC na saída superior, o que também é observado na realidade. Ciclones acoplados a esta saída são necessários para promover o retorno destes sólidos ao processo. Aglomerados podem ser identificados em algumas regiões ao longo do secador. Estes podem ser visualizados em cores no plano $\mathrm{XZ}$ em $\mathrm{y}=0,5 \mathrm{~m}$ da Figura 4 e representam um problema na realidade, devido à sua contribuição para a ineficiência do processo de secagem.

Outras previsões do modelo invíscido (não mostradas) foram zonas de recirculação de ar devido à interação do sólido com o gás, bem como um maior gradiente de pressão na região inferior, e maiores gradientes de velocidade tanto para o sólido como para a fase contínua próximos às saídas.

Com a teoria cinética do escoamento de materiais granulares não foi possível prever o padrão de escoamento do leito fluidizado, ficando todo o sólido na região inferior do secador conforme mostra a Figura 5. 


\section{9 a 22 de outubro de 2014 \\ Florianópolis/SC}

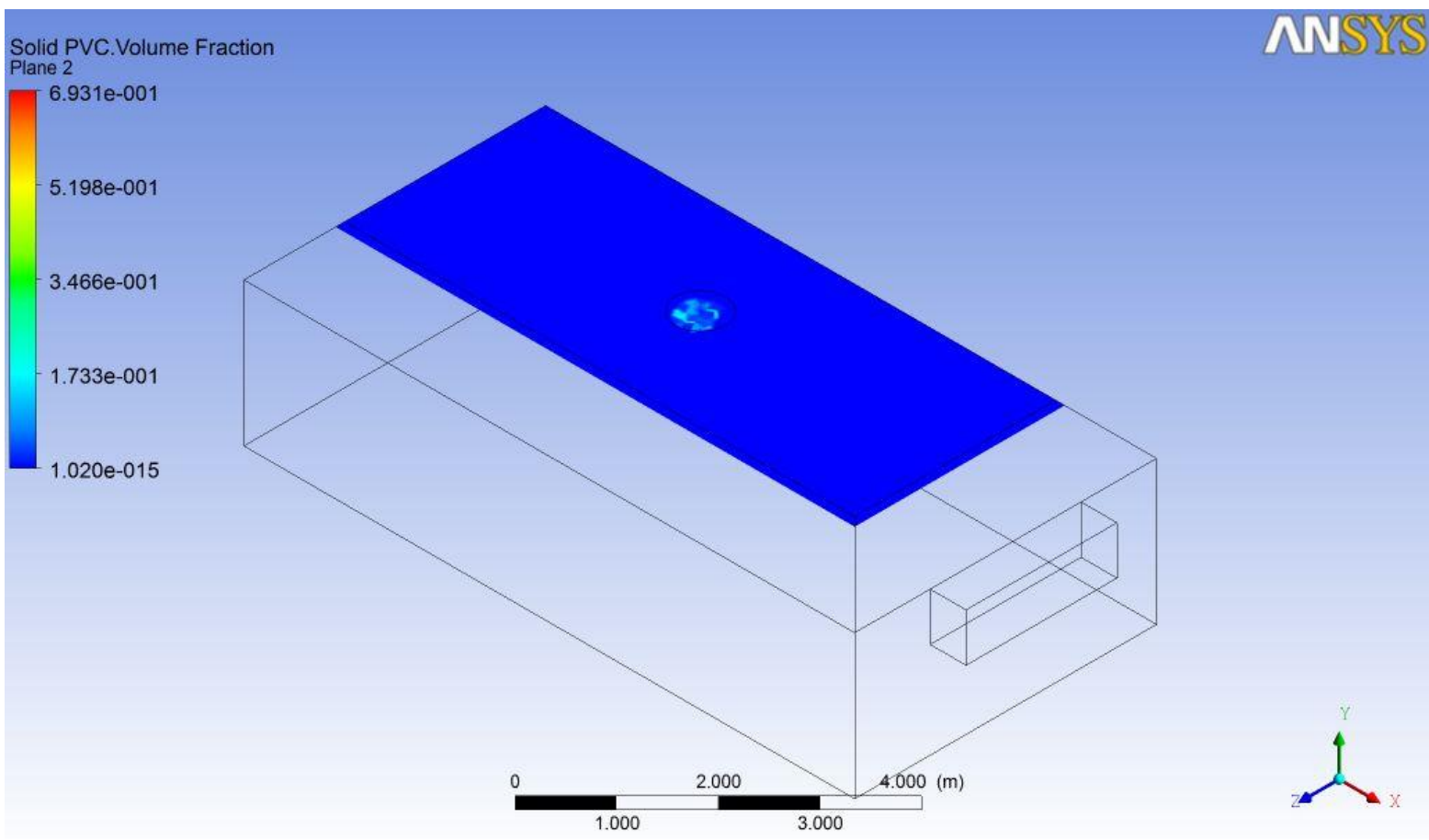

Figura 3 - Pequena fração de PVC na saída superior para o modelo invíscido.

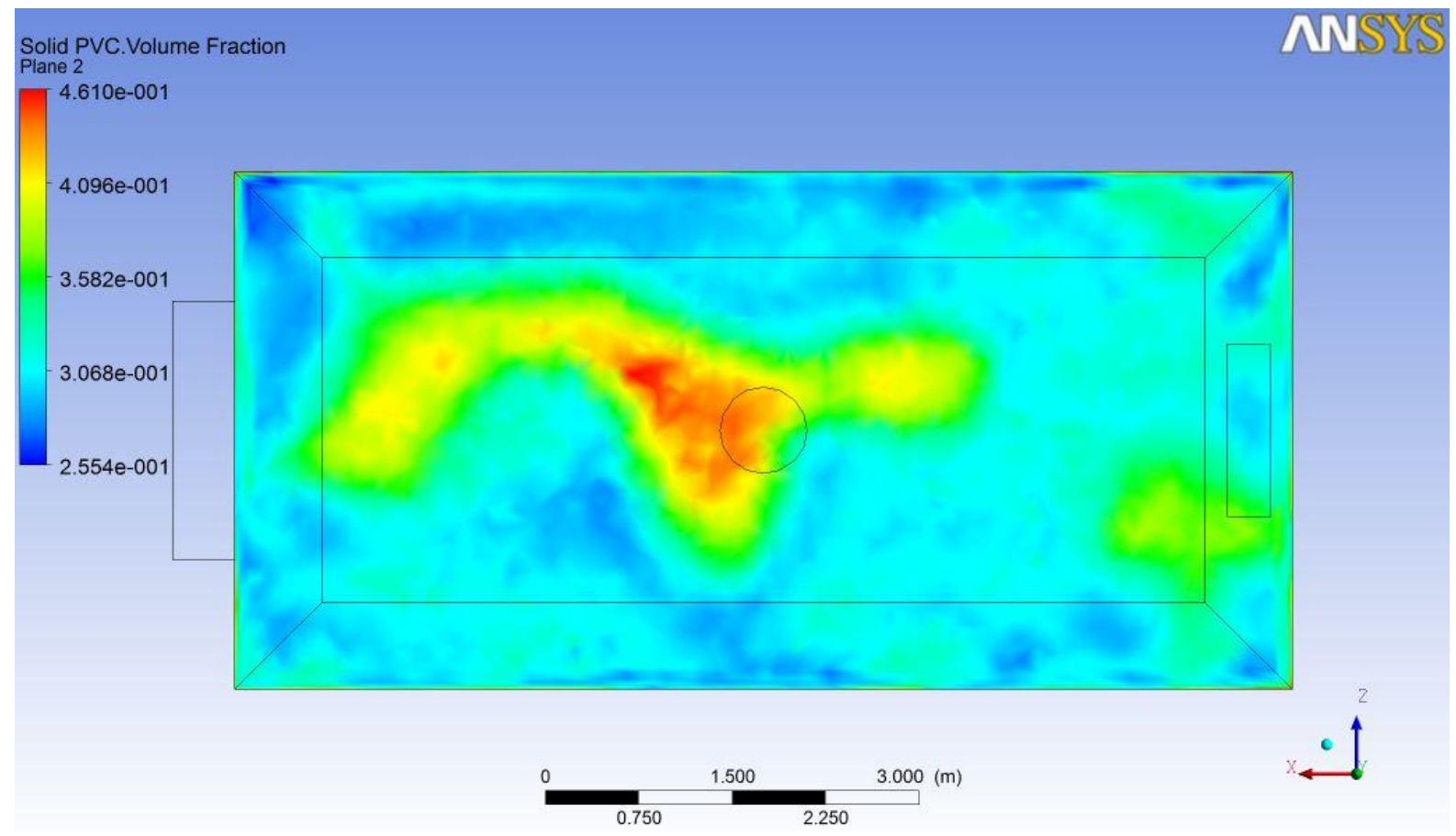

Figura 4 - Formação de clusters no plano XZ em y = 5 m para o modelo invíscido. 


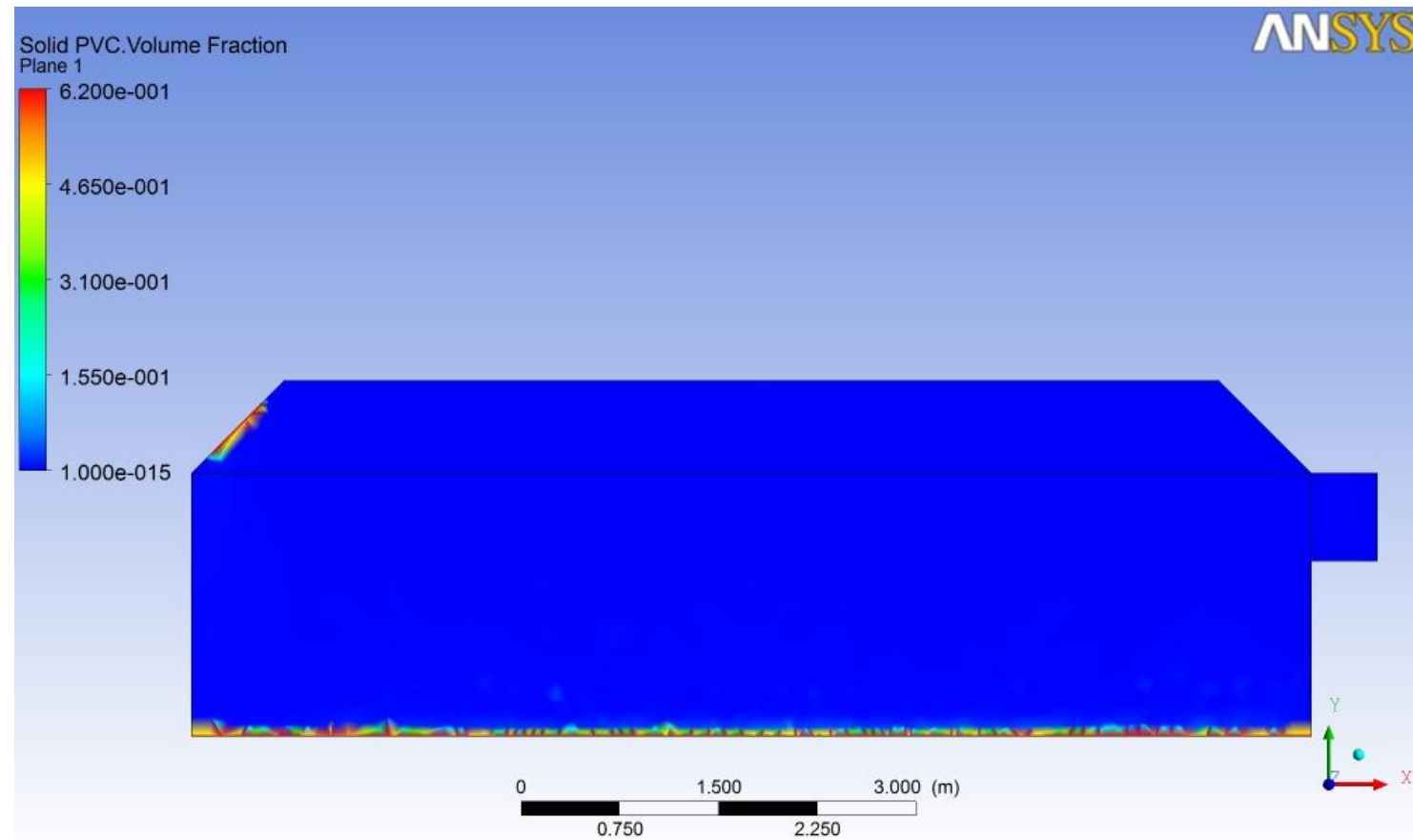

Figura 5 - Fração volumétrica no plano XY em z $=2 \mathrm{~m}$ para o modelo que considera a teoria cinética.

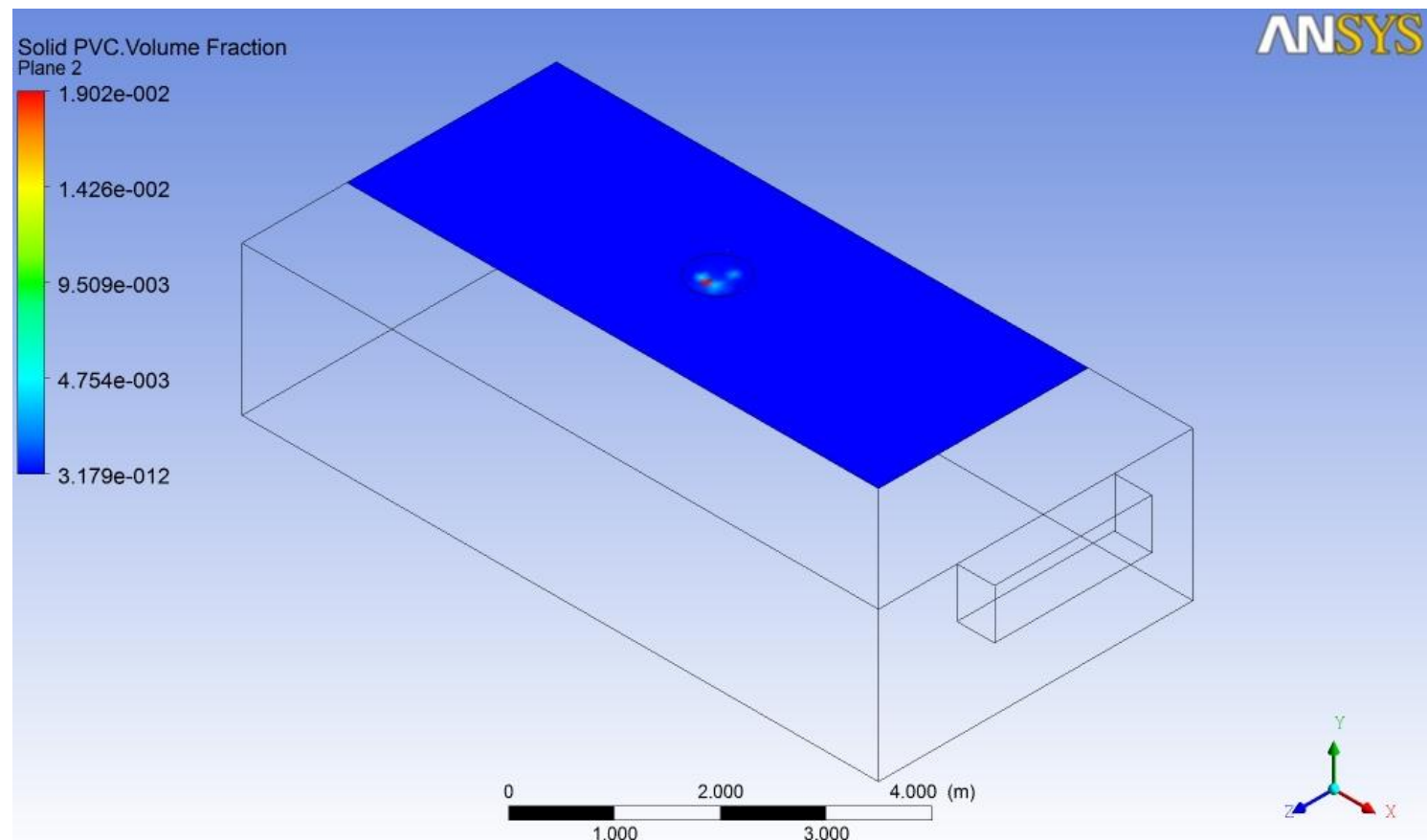

Figura 6 - Pequena fração de PVC na saída superior para o modelo que considera a teoria cinética. 


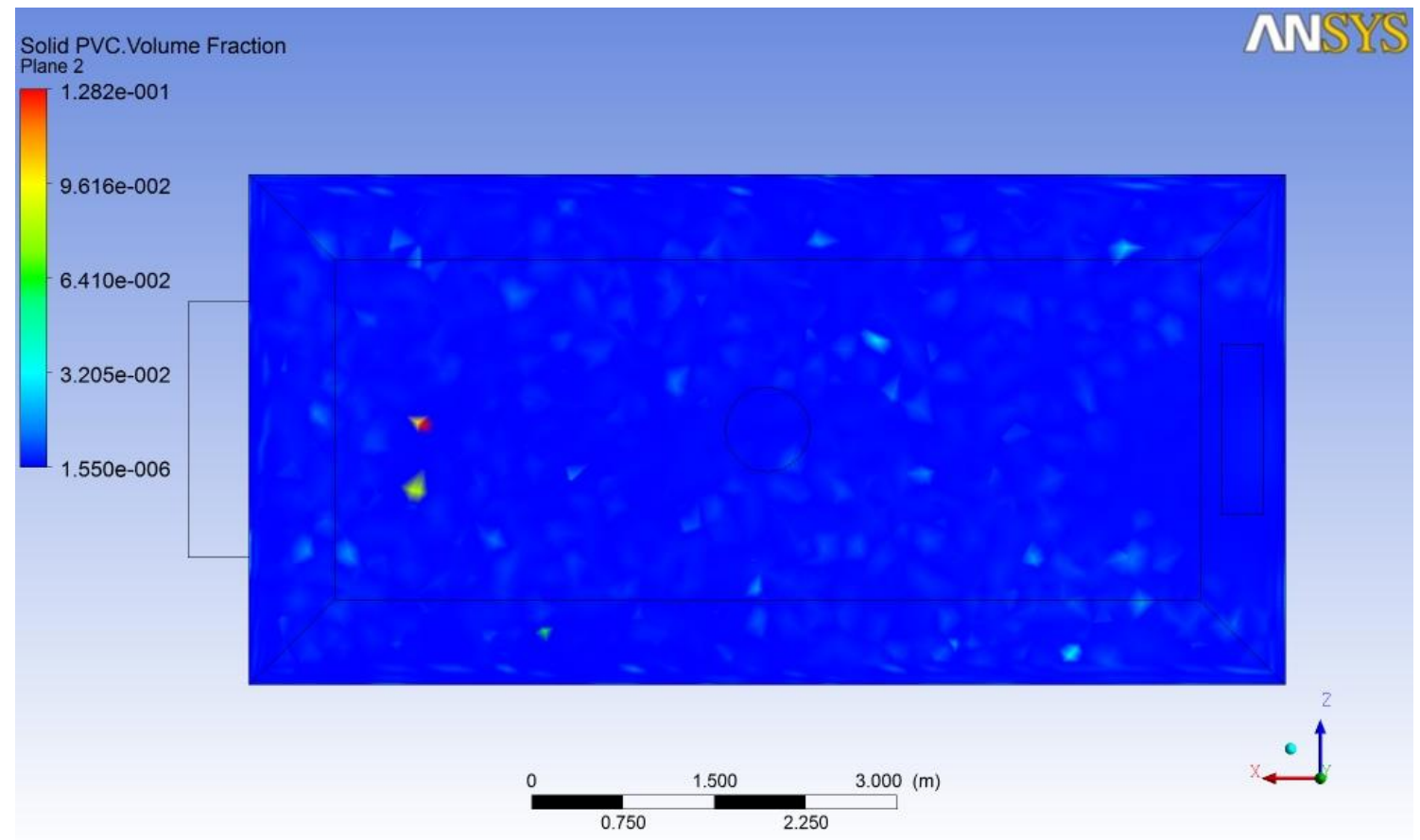

Figura 7 - Formação de clusters no plano XZ em y =0,5 m para o modelo que considera a teoria cinética.

Mesmo sem a formação do leito, é possível identificar que uma pequena fração de sólidos é arrastada pela saída superior do secador, porém por uma região menor do que para o modelo invíscido, o que pode ser visualizado na Figura 6. Menores aglomerados foram previstos com o uso da KTGF em relação ao modelo invíscido, conforme mostrado na Figura 7.

\section{CONCLUSÃO}

Os resultados mostraram que técnicas de CFD são capazes de prever o padrão de escoamento existente em secadores de leito fluidizado. O modelo de fluido invíscido para a fase particulada foi capaz de prever a formação do leito de partículas no interior do secador. Já com o modelo da KTGF, esta característica do leito não foi prevista.

A simulação com o modelo invíscido proporcionou a identificação de características típicas da operação deste secador tais como a formação de aglomerados e a presença de zonas de recirculação. Porém o modelo da KTGF não foi capaz de prever os padrões de escoamento para o secador industrial estudado, nem a formação do leito fluidizado. Este último necessita de vários parâmetros, como o coeficiente de restituição partícula-partícula, limite máximo para a temperatura granular, entre outros. O ajuste destes parâmetros é de fundamental importância para uma correta previsão com o modelo da KTGF. Convém salientar, também, que o modelo da KTGF utilizado foi o padrão do software CFX, que é um modelo simplificado de equilíbrio. Estes dois últimos fatores podem justificar a não 
previsão do padrão de escoamento com o uso do modelo da KTGF.

Com base neste estudo, trabalhos futuros podem surgir com ênfase em otimização, com o propósito de minimizar a formação de aglomerados. Novas geometrias deste secador podem ser sugeridas e estudadas para que uma melhor eficiência de secagem com menores custos seja obtida.

\section{AGRADECIMENTOS}

Os autores agradecem ao incentivo financeiro provido pelo FNDE/PET, ao DEQ/UFCG e ao Laboratório de Experimentação Numérica de Processos (LENP) da Unidade Acadêmica de Engenharia Química - Universidade Federal de Campina Grande.

\section{REFERÊNCIAS}

ALVES, J. J. N. Modelagem fenomenológica e simulação bidimensional da fluidodinâmica de reatores de leito fluidizado circulante. Tese de doutorado, Universidade Estadual de Campinas/São Paulo,1998.

ANSYS-CFX release 11.0 Reference manual, 2011.

ARAUJO, A. C. B.; VASCONCELOS, L. G. S.; ALVES, J. J. N.; SHANG, H. Optimal operation of an industrial PVC dryer. Dry. Tech., v. 29, p. 19-34, 2010.

ASSARI, M. R.; TABRIZI, H. B.; NAJAFPOUR, E. Energy and exergy analysis of fluidized bed dryer based on two-fluid modeling. Int. J. of Therm. Sc., v. 64, p. 213-219, 2013.

COUTINHO, S. A. Modelagem e simulação de reatores de craqueamento catalítico do petróleo utilizando o software CFX. Dissertação de mestrado, Universidade Federal de Campina Grande/Paraíba, 2003.

GOLDSCHMIDT, M. J. V.; BEETSTRA, R.; KUIPERS, J. A. M. Hydrodynamic modelling of dense gas-fluidised beds: comparison of the kinetic theory of granular flow with 3D hard-sphere discrete particle simulations. Chem. Eng. Sc., v. 57, p. 2059-2075, 2002.

NIEUWLAND, J. J.; VAN SINT ANNALAND, KUIPERS, M. M.; VAN SWAAIJ, W. P. M. Hydrodynamic modeling of gas particle flows in riser reactors. AIChE J., v. 42, n.6, p 1569 1582, 1996.

TACIDELli, A. R.; PEREIRA NETO, A. T.; BRITO, R. P.; ARAUJO, A. C. B.; VASCONCELOS, L. G. S.; ALVES, J. J. N. Modeling and simulation of industrial PVC drying in fluidized beds with internal heat source. Chem. Eng. \& Tech. Dry., v. 35, n. 12, p. 2107 - 2119, 2012.

VAN DER HOEF, M. A.; VAN SINT ANNALAND, M.; KUIPERS, J. A. M. Computational fluid dynamics for dense gas-solid fluidized beds: a multi-scale modeling strategy. China Part., v. 3, n. 1-2, p. 69-77, 2005. 